\title{
SPECTRALLY NEGATIVE LÉVY PROCESSES PERTURBED BY FUNCTIONALS OF THEIR RUNNING SUPREMUM
}

\author{
ANDREAS E. KYPRIANOU *** AND \\ CURDIN OTT, ${ }^{*}$ University of Bath
}

\begin{abstract}
In the setting of the classical Cramér-Lundberg risk insurance model, Albrecher and Hipp (2007) introduced the idea of tax payments. More precisely, if $X=$ $\left\{X_{t}: t \geq 0\right\}$ represents the Cramér-Lundberg process and, for all $t \geq 0, S_{t}:=\sup _{s \leq t} X_{s}$, then Albrecher and Hipp studied $X_{t}-\gamma S_{t}, t \geq 0$, where $\gamma \in(0,1)$ is the rate at which tax is paid. This model has been generalised to the setting that $X$ is a spectrally negative Lévy process by Albrecher, Renaud and Zhou (2008). Finally, Kyprianou and Zhou (2009) extended this model further by allowing the rate at which tax is paid with respect to the process $S=\left\{S_{t}: t \geq 0\right\}$ to vary as a function of the current value of $S$. Specifically, they considered the so-called perturbed spectrally negative Lévy process, $U_{t}:=X_{t}-\int_{(0, t]} \gamma\left(S_{u}\right) \mathrm{d} S_{u}, t \geq 0$, under the assumptions that $\gamma:[0, \infty) \rightarrow[0,1)$ and $\int_{0}^{\infty}(1-\gamma(s)) \mathrm{d} s=\infty$. In this article we show that a number of the identities in Kyprianou and Zhou (2009) are still valid for a much more general class of rate functions $\gamma:[0, \infty) \rightarrow \mathbb{R}$. Moreover, we show that, with appropriately chosen $\gamma$, the perturbed process can pass continuously (i.e. creep) into $(-\infty, 0)$ in two different ways.
\end{abstract}

Keywords: Spectrally negative Lévy process; excursion theory; creeping; ruin

2010 Mathematics Subject Classification: Primary 60K05; 60K15; 91B30

\section{Introduction}

Let $X=\left\{X_{t}: t \geq 0\right\}$ be a spectrally negative Lévy process defined on a filtered probability space $\left(\Omega, \mathcal{F}, \mathbb{F}=\left\{\mathcal{F}_{t}\right\}_{t \geq 0}, \mathrm{P}\right)$ satisfying the natural conditions (cf. Section 1.3 of [5, p. 39]); that is to say, a one-dimensional process which has stationary and independent increments, and càdlàg paths with only negative discontinuities, but which does not have monotone paths. For $x \in \mathbb{R}$, denote by $\mathrm{P}_{x}$ the probability measure under which $X$ starts at $x$ and write $\mathrm{P}_{0}=\mathrm{P}$. It is well known that a spectrally negative Lévy process $X$ is characterised by its Lévy triplet $(b, \sigma, \Pi)$, where $\sigma \geq 0, b \in \mathbb{R}$, and $\Pi$ is a measure on $(-\infty, 0)$ satisfying the condition $\int_{(-\infty, 0)}\left(1 \wedge x^{2}\right) \Pi(\mathrm{d} x)<\infty$. By the Lévy-Itô decomposition, $X$ may be represented in the form

$$
X_{t}=\sigma B_{t}-b t+X_{t}^{(1)}+X_{t}^{(2)},
$$

where $\left\{B_{t}: t \geq 0\right\}$ is a standard Brownian motion, $\left\{X_{t}^{(1)}: t \geq 0\right\}$ is a compound Poisson process with discontinuities of magnitude bigger than or equal to 1 , and $\left\{X_{t}^{(2)}: t \geq 0\right\}$ is a squareintegrable martingale with discontinuities of magnitude strictly smaller than 1 , with the three

Received 17 April 2012; revision received 31 May 2012.

* Postal address: Department of Mathematical Sciences, University of Bath, Claverton Down, Bath, BA2 7AY, UK.

** Email address: a.kyprianou @bath.ac.uk 
processes mutually independent. In particular, if $X$ is of bounded variation, the decomposition reduces to

$$
X_{t}=\mathrm{d} t-\eta_{t}
$$

where $\mathrm{d}>0$ and $\left\{\eta_{t}: t \geq 0\right\}$ is a driftless subordinator. Furthermore, let

$$
\psi(\theta):=\log \mathrm{E}\left[\mathrm{e}^{\theta X_{1}}\right], \quad \theta \geq 0,
$$

be the Laplace exponent of $X$ which is known to be a strictly convex and infinitely differentiable function on $[0, \infty)$. The asymptotic behavior of $X$ is characterised by $\psi^{\prime}(0+)$, so that $X$ drifts to $\pm \infty$ or oscillates according to whether $\pm \psi^{\prime}(0+)>0$ or, respectively, $\psi^{\prime}(0+)=0$.

Denote by $S=\left\{S_{t}: t \geq 0\right\}$ the running supremum, that is, $S_{t}:=\sup _{s \leq t} X_{s}$ for each $t \geq 0$. We are interested in perturbing $X$ by some functional of its running supremum $S$. Motivated by the results in [7], our primary object of study is given by $U=\left\{U_{t}: t \geq 0\right\}$, where

$$
U_{t}:=X_{t}-\int_{(0, t]} \gamma\left(S_{u}\right) \mathrm{d} S_{u}, \quad t \geq 0,
$$

for some locally integrable function $\gamma:[0, \infty) \rightarrow \mathbb{R}$. Such processes have appeared in the context of insurance risk models with tax, where $X$ plays the role of the so-called surplus process (the wealth of an insurance company) and $\gamma$ characterises the rate at which tax is paid with respect to the running maximum. One may also think of the process $U$ as a spectrally negative Lévy process perturbed by a functional of its maximum in the spirit of [9]. In the special case that $\gamma:[0, \infty) \rightarrow[0,1)$ and $\int_{0}^{\infty}(1-\gamma(s)) \mathrm{d} s=\infty$, our process $U$ agrees with the process studied in [7]. Under the even stronger assumption that $\gamma$ is a constant in $(0,1)$, the resulting process has been considered in [1] and [2]. In the simple case that $\gamma=0$ we are back to the process $X$, the so-called Lévy insurance risk process in the context of ruin theory. The main objective of this article is to show that all of the identities in [7] carry over to the setting where $\gamma$ belongs to the general class of locally integrable functions. Moreover, we will show that, for some choices of $\gamma$, it is possible for the process $U$ to enter $(-\infty, 0)$ continuously in two different ways.

The key observation which, with the help of excursion theory, leads to all our results is that we may write $U$ in the form

$$
U_{t}=A_{t}-\left(S_{t}-X_{t}\right), \quad t \geq 0,
$$

where the process $A=\left\{A_{t}: t \geq 0\right\}$ is given by

$$
A_{t}:=S_{t}-\int_{(0, t]} \gamma\left(S_{u}\right) \mathrm{d} S_{u}, \quad t \geq 0 .
$$

Assuming that $X_{0}=x$, we may write $A_{t}=\bar{\gamma}\left(S_{t}\right)$, where

$$
\bar{\gamma}(s):=s-\int_{x}^{s} \gamma(y) \mathrm{d} y, \quad s \geq x .
$$

Note that $A$ is a process of bounded variation and accordingly we may think of $\mathrm{d} A$ as a signed measure whose support, say $\mathcal{A}$, is contained in the support of the measure d $S$. Suppose now that $\mathscr{B}$ consists of the countable union of open intervals of time which correspond to the epochs that the process $S-X$ spends away from 0 . Then $\mathcal{A} \cap \mathscr{B}=\varnothing$. As a consequence, 

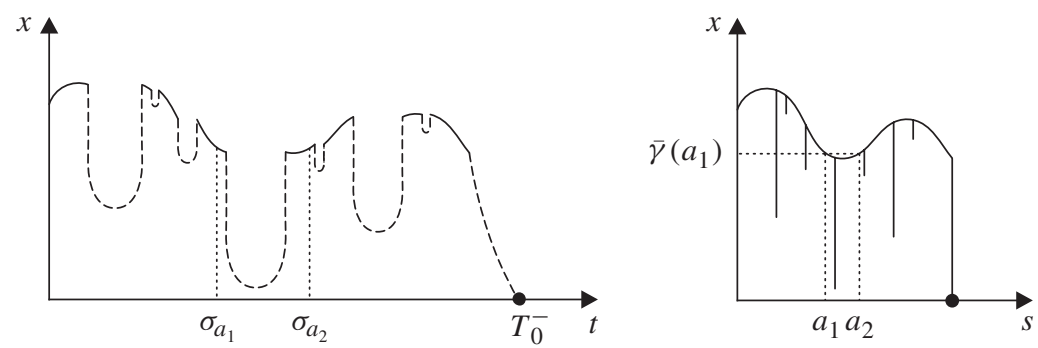

FIGURE 1: A symbolic realisation of the trajectory of $U$ up to the moment it first enters $(-\infty, 0)$, with its corresponding trace in the $(x, s)$-plane. The dashed $\mathrm{u}$-shaped curves are schematic representations of excursions of $X$ away from its maximum or, put differently, excursions of $U$ away from $A$.

we may interpret (1.2) as a path decomposition in which excursions of $X$ from its maximum (equivalently, excursions of $S-X$ away from 0 ) are 'hung' off the trajectory of $A$ between its increment times (see Figure 1 for a symbolic representation). A more detailed description of this excursion-theoretic decomposition will follow in due course.

We conclude this section by introducing the so-called scale functions (cf. [6] and/or [8]) which will henceforth play an important role and are defined as follows. For each given $q \geq 0$, we have $W^{(q)}(x)=0$ when $x<0$, and otherwise on $[0, \infty), W^{(q)}$ is the unique right-continuous function whose Laplace transform is

$$
\int_{0}^{\infty} \mathrm{e}^{-\theta x} W^{(q)}(x) \mathrm{d} x=\frac{1}{\psi(\theta)-q}, \quad \theta>\Phi(q),
$$

where $\Phi(q)$ is the largest solution to the equation $\psi(\theta)=q$ (there are at most two). For notational convenience, we will write $W^{(0)}=W$. It is shown in Lemma 2.3 of [6] that, for any $q \geq 0, W^{(q)}$ is absolutely continuous with respect to the Lebesgue measure and strictly increasing. If $X$ is of unbounded variation, it is additionally known that $W^{(q)}$ is continuously differentiable on $(0, \infty)$ (cf. Lemma 2.4 of [6]). In either case we will denote by $W^{(q)^{\prime}}$ the associated density whenever it appears in a Lebesgue integral. Finally, the behavior of $W^{(q)}$ and its right derivative, written $W_{+}^{(q) \prime}$, at 0 are known. Specifically, for all $q \geq 0$, we have

$$
W^{(q)}(0+)= \begin{cases}d^{-1} & \text { if } X \text { is of bounded variation, } \\ 0 & \text { if } X \text { is of unbounded variation, }\end{cases}
$$

and

$$
W_{+}^{(q)^{\prime}}(0+)= \begin{cases}\frac{q+\Pi(-\infty, 0)}{\mathrm{d}^{2}} & \text { if } \sigma=0 \text { and } \Pi(-\infty, 0)<\infty, \\ \frac{2}{\sigma^{2}} & \text { if } \sigma>0 \text { or } \Pi(-\infty, 0)=\infty,\end{cases}
$$

where we understand the second case to be $+\infty$ when $\sigma=0$ (cf. Lemmas 3.1 and 3.2 of [6]).

\section{Results}

Let us introduce

$$
\sigma_{a}:=\inf \left\{t>0: S_{t}=a\right\} \quad \text { and } \quad T_{0}^{-}:=\inf \left\{t>0: U_{t}<0\right\},
$$

where we use the usual convention that inf $\varnothing:=\infty$. 
Theorem 2.1. (One-sided and two-sided exit formulae.) Let $x>0$ be given, and define $a^{*}(x):=\inf \{s \in[x, \infty): \bar{\gamma}(s)<0\} \in(x, \infty]$, the maximal value $S$ can possibly attain up to time $T_{0}^{-}$. Then, for any $q \geq 0$ and $x \leq a<a^{*}(x)$, the following statements hold.

(a)

$$
\mathrm{E}_{x}\left[\mathrm{e}^{-q \sigma_{a}} \mathbf{1}_{\left\{\sigma_{a}<T_{0}^{-}\right\}}\right]=\exp \left(-\int_{x}^{a} \frac{W^{(q) \prime}(\bar{\gamma}(s))}{W^{(q)}(\bar{\gamma}(s))} \mathrm{d} s\right)
$$

(b)

$$
\mathrm{E}_{x}\left[\mathrm{e}^{-q T_{0}^{-}} \mathbf{1}_{\left\{T_{0}^{-}<\sigma_{a}\right\}}\right]=\int_{x}^{a} \exp \left(-\int_{x}^{t} \frac{W^{(q) \prime}(\bar{\gamma}(s))}{W^{(q)}(\bar{\gamma}(s))} \mathrm{d} s\right) f(\bar{\gamma}(t)) \mathrm{d} t,
$$

where

$$
f(z)=\frac{Z^{(q)}(z) W^{(q) \prime}(z)}{W^{(q)}(z)}-q W^{(q)}(z)
$$

and $Z^{(q)}(z):=1+q \int_{0}^{z} W^{(q)}(y) \mathrm{d} y$.

(c) Suppose that $a^{*}(x)=\infty$. Then

$$
\mathrm{E}_{x}\left[\mathrm{e}^{-q T_{0}^{-}} \mathbf{1}_{\left\{T_{0}^{-}<\infty\right\}}\right]=\int_{x}^{\infty} \exp \left(-\int_{x}^{t} \frac{W^{(q) \prime}(\bar{\gamma}(s))}{W^{(q)}(\bar{\gamma}(s))} \mathrm{d} s\right) f(\bar{\gamma}(t)) \mathrm{d} t .
$$

In particular,

$$
\mathrm{P}_{x}\left[T_{0}^{-}<\infty\right]=1-\exp \left(-\int_{x}^{\infty} \frac{W^{\prime}(\bar{\gamma}(s))}{W(\bar{\gamma}(s))} \mathrm{d} s\right) .
$$

Remark 2.1. If we assume that $\gamma:[0, \infty) \rightarrow[0,1)$ with $\int_{0}^{\infty}(1-\gamma(s)) \mathrm{d} s=\infty$, then $\bar{\gamma}$ is continuous, strictly increasing, and has a well-defined inverse on $[x, \infty)$ which we will denote by $\bar{\gamma}^{-1}$. Then, for $a \geq x$, if we write $T_{a}^{+}:=\inf \left\{t>0: U_{t}>a\right\}=\sigma_{\bar{\gamma}^{-1}(a)}$, Theorem 2.1 reads

$$
\begin{aligned}
\mathrm{E}_{x}\left[\mathrm{e}^{-q T_{a}^{+}} \mathbf{1}_{\left\{T_{a}^{+}<T_{0}^{-}\right\}}\right] & =\exp \left(-\int_{x}^{\bar{\gamma}^{-1}(a)} \frac{W^{(q) \prime}(\bar{\gamma}(s))}{W^{(q)}(\bar{\gamma}(s))} \mathrm{d} s\right) \\
& =\exp \left(-\int_{x}^{a} \frac{W^{(q) \prime}(y)}{W^{(q)}(y)\left(1-\gamma\left(\bar{\gamma}^{-1}(y)\right)\right)} \mathrm{d} y\right),
\end{aligned}
$$

which agrees with Theorem 1.1 of [7]. Similarly, if $\gamma=0$ then $U_{t}=X_{t}$ and $\tau_{a}^{+}:=$ $\inf \left\{t>0: X_{t}>a\right\}$, and Theorem 2.1 reduces to

$$
\mathrm{E}_{x}\left[\mathrm{e}^{-q \tau_{a}^{+}} \mathbf{1}_{\left\{\tau_{a}^{+}<T_{0}^{-}\right\}}\right]=\exp \left(-\int_{x}^{a} \frac{W^{(q) \prime}(s)}{W^{(q)}(s)} \mathrm{d} s\right)=\frac{W^{(q)}(x)}{W^{(q)}(a)}
$$

and

$$
\begin{aligned}
& \mathrm{E}_{x}\left[\mathrm{e}^{-q T_{0}^{-}} \mathbf{1}_{\left\{T_{0}^{-}<\tau_{a}^{+}\right\}}\right]=\int_{x}^{a} \frac{W^{(q)}(x)}{W^{(q)}(t)} f(t) \mathrm{d} t \\
& =-W^{(q)}(x) \int_{x}^{a}\left(\frac{Z^{(q)}}{W^{(q)}}\right)^{\prime}(t) \mathrm{d} t \\
& =Z^{(q)}(x)-W^{(q)}(x) \frac{Z^{(q)}(a)}{W^{(q)}(a)},
\end{aligned}
$$

where $Z^{(q)}(x):=1+q \int_{0}^{x} W^{(q)}(y) \mathrm{d} y$. This agrees with Equations (8.8) and (8.9) of [8]. Also, by a straightforward calculation we see that (2.2) and (2.3) reduce to Equations (8.6) and (8.7) of [8]. 
Remark 2.2. Fix $x>0$, and suppose that $\gamma(s) \equiv \gamma \in(1, \infty)$. It follows that $\bar{\gamma}(s)=$ $s(1-\gamma)+\gamma x$ and $a^{*}(x)=\gamma x /(\gamma-1)$. Then, for $q \geq 0$ and $x \leq a<a^{*}(x)$, the expression in (2.1) simplifies to

$$
\begin{aligned}
\mathrm{E}_{x}\left[\mathrm{e}^{-q \sigma_{a}} \mathbf{1}_{\left\{\sigma_{a}<T_{0}^{-}\right\}}\right] & =\exp \left(\frac{1}{1-\gamma} \int_{a(1-\gamma)+\gamma x}^{x} \frac{W^{(q) \prime}(u)}{W^{(q)}(u)} \mathrm{d} u\right) \\
& =\left(\frac{W^{(q)}(a(1-\gamma)+\gamma x)}{W^{(q)}(x)}\right)^{1 /(\gamma-1)} .
\end{aligned}
$$

Moreover, if $\gamma(s) \equiv \gamma \in(0,1)$, we may recover the first formula in Remark 1.1 of [7] by a similar computation or an application of (2.4).

Remark 2.3. Let $x>0$ be given, and assume that $X$ drifts to $+\infty$ or, equivalently, that $\psi^{\prime}(0+)>0$. Moreover, suppose that $\gamma(s) \equiv \gamma \in(0,1)$ and, hence, $\bar{\gamma}(s)=s(1-\gamma)+\gamma x$. Then, using the fact that $\lim _{s \rightarrow \infty} W(s)=1 / \psi^{\prime}(0+)$ (cf. Lemma 3.3 of [6]), it follows from (2.3) that

$$
\mathrm{P}_{x}\left[T_{0}^{-}<\infty\right]=1-\exp \left(-\frac{1}{1-\gamma} \int_{x}^{\infty} \frac{W^{\prime}(s)}{W(s)} \mathrm{d} s\right)=1-\left(\psi^{\prime}(0+) W(x)\right)^{1 /(1-\gamma)} .
$$

This is analogous to Equation (8.7) of [8].

The proof of Theorem 2.1 makes heavy use of excursion theory for the process $S-X$. We refer the reader to [4, Chapters 6 and 7] for background reading. We will spend a moment setting up some necessary notation which will be used throughout the remainder of the paper. The process $L_{t}:=S_{t}-x$ serves as a local time at 0 for the Markov process $S-X$ under $\mathrm{P}_{x}$. Write $L^{-1}:=\left\{L_{t}^{-1}: t \geq 0\right\}$ for the right-continuous inverse of $L$. The Poisson point process of excursion indexed by the local time will be denoted by $\left\{\left(t, \varepsilon_{t}\right): t \geq 0\right\}$, where

$$
\varepsilon_{t}:=\left\{\varepsilon_{t}(s):=X_{L_{t}^{-1}}-X_{L_{t-}^{-1}+s}: 0<s<L_{t}^{-1}-L_{t-}^{-1}\right\}
$$

whenever $L_{t}^{-1}-L_{t-}^{-1}>0$. Accordingly, we refer to a generic excursion as $\varepsilon(\cdot)$ (or just $\varepsilon$ for short) belonging to the space $\mathcal{E}$ of canonical excursions. The intensity measure of the process $\left\{\left(t, \varepsilon_{t}\right): t \geq 0\right\}$ is given by $\mathrm{d} t \times \mathrm{d} n$, where $n$ is a measure on the space of excursions (the excursion measure). A functional of the canonical excursion which will be of interest is $\bar{\varepsilon}:=\sup _{s<\zeta} \varepsilon(s)$, where $\zeta(\varepsilon)=\zeta$ is the length of an excursion. A useful formula for this functional that we will make use of is (cf. [8, Equation (8.18)])

$$
n(\bar{\varepsilon}>x)=\frac{W^{\prime}(x)}{W(x)},
$$

provided that $x$ is not a discontinuity point in the derivative of $W$, which is only a concern when $X$ is of bounded variation, in which case there are at most countably many such discontinuities. Another functional of $\varepsilon$ that we will also use is $\rho_{k}:=\inf \{s>0: \varepsilon(s)>k\}$, the first passage time above $k$ of the canonical excursion $\varepsilon$. Note that, for $a \geq x$, it follows that, under $\mathrm{P}_{x}$, the event that $S_{t}=a$ coincides with the event that the process $S_{t}$ climbs from $x$ to $a$ for the first time. Consequently, $L_{a-x}^{-1}=\tau_{a}^{+}$.

Proof of Theorem 2.1. (a) For $a \geq x$, we have $\left\{\sigma_{a}<T_{0}^{-}\right\}=\left\{\bar{\varepsilon}_{s} \leq \bar{\gamma}(x+s)\right.$ for all $0 \leq s \leq a-x\}$. Recall that, for each $q \geq 0$, we have the exponential change of measure

$$
\left.\frac{\mathrm{dP}^{\Phi(q)}}{\mathrm{dP}}\right|_{\left\{X_{s}: s \leq t\right\}}=\mathrm{e}^{\Phi(q) X_{t}-q t}, \quad t \geq 0 .
$$


Then, recalling that, for each $t \geq 0, L_{t}^{-1}$ is a stopping time, we have, for $x>0$,

$$
\begin{aligned}
\mathrm{E}_{x}\left[\mathrm{e}^{-q \sigma_{a}} \mathbf{1}_{\left\{\sigma_{a}<T_{0}^{-}\right\}}\right] & =\mathrm{E}_{x}\left[\mathrm{e}^{-q L_{a-x}^{-1}} \mathbf{1}_{\left\{\bar{\varepsilon}_{s} \leq \bar{\gamma}(x+s) \text { for all } 0 \leq s \leq a-x\right\}}\right] \\
& =\mathrm{e}^{-(a-x) \Phi(q)} \mathrm{E}_{x}^{\Phi(q)}\left[\mathbf{1}_{\left\{\bar{\varepsilon}_{s} \leq \bar{\gamma}(x+s) \text { for all } 0 \leq s \leq a-x\right\}}\right] \\
& =\mathrm{e}^{-(a-x) \Phi(q)} \exp \left(-\int_{0}^{a-x} n_{\Phi(q)}(\bar{\varepsilon}>\bar{\gamma}(x+s) \mathrm{d} s)\right) \\
& =\exp \left(-\int_{0}^{a-x} \frac{W^{(q) \prime}(\bar{\gamma}(x+s))}{W^{(q)}(\bar{\gamma}(x+s))} \mathrm{d} s\right) .
\end{aligned}
$$

Here, $n_{\Phi(q)}$ is the excursion measure of $S-X$ under $\mathrm{P}^{\Phi(q)}$, which is known to satisfy

$$
n_{\Phi(q)}(\bar{\varepsilon}>x)=\frac{W^{(q) \prime}(x)}{W^{(q)}(x)}-\Phi(q) ;
$$

see, for example, Formula (2.7) of [7]. Now changing variables in (2.5) gives (2.1).

(b) An application of the compensation formula yields

$$
\begin{aligned}
\mathrm{E}_{x}\left[\mathrm{e}^{-q T_{0}^{-}} \mathbf{1}_{\left\{T_{0}^{-}<\sigma_{a}\right\}}\right] & \\
& =\mathrm{E}_{x}\left[\sum_{0<t \leq a-x} \mathrm{e}^{-q L_{t-}^{-1}-q \rho_{\bar{\gamma}(t+x)}\left(\varepsilon_{t}\right)} \mathbf{1}_{\left\{\bar{\varepsilon}_{s} \leq \bar{\gamma}(s+x) \text { for all } s<t\right\}} \mathbf{1}_{\left\{\bar{\varepsilon}_{t}>\bar{\gamma}(t+x)\right\}}\right] \\
& =\mathrm{E}_{x}\left[\int_{0}^{a-x} \mathrm{e}^{-q L_{t}^{-1}} \mathbf{1}_{\left\{\bar{\varepsilon}_{s} \leq \bar{\gamma}(s+x) \text { for all } s<t\right\}} \int_{\mathcal{E}} \mathrm{e}^{-q \rho_{\bar{\gamma}(t+x)}(\varepsilon)} \mathbf{1}_{\{\bar{\varepsilon}>\bar{\gamma}(t+x)\}} n(\mathrm{~d} \varepsilon) \mathrm{d} t\right] \\
& =\mathrm{E}_{x}\left[\int_{0}^{a-x} \mathrm{e}^{-q L_{t}^{-1}} \mathbf{1}_{\left\{\bar{\varepsilon}_{s} \leq \bar{\gamma}(s+x) \text { for all } s<t\right\}} f(\bar{\gamma}(t+x)) \mathrm{d} t\right] \\
& =\int_{0}^{a-x} \mathrm{e}^{-t \Phi(q)} \mathrm{E}_{x}^{\Phi(q)}\left[\mathbf{1}_{\left\{\bar{\varepsilon}_{s} \leq \bar{\gamma}(s+x) \text { for all } s<t\right\}}\right] f(\bar{\gamma}(t+x)) \mathrm{d} t \\
& =\int_{0}^{a-x} \mathrm{e}^{-t \Phi(q)} \exp \left(-\int_{0}^{t} n_{\Phi(q)}(\bar{\varepsilon}>\bar{\gamma}(s+x)) \mathrm{d} s\right) f(\bar{\gamma}(t+x)) \mathrm{d} t \\
& =\int_{0}^{a-x} \exp \left(-\int_{0}^{t} \frac{W^{(q) \prime}(\bar{\gamma}(s+x))}{W^{(q)}(\bar{\gamma}(s+x))} \mathrm{d} s\right) f(\bar{\gamma}(t+x)) \mathrm{d} t,
\end{aligned}
$$

where in the first equality the time index runs over local times and the sum is the usual shorthand for integration with respect to the Poisson counting measure of excursions, and

$$
f(z):=\int_{\mathcal{E}} \mathrm{e}^{-q \rho_{z}(\varepsilon)} \mathbf{1}_{\{\bar{\varepsilon}>z\}} n(\mathrm{~d} \varepsilon)=\frac{Z^{(q)}(z) W^{(q) \prime}(z)}{W^{(q)}(z)}-q W^{(q)}(z)
$$

is an expression taken from Theorem 1 (Equation (18)) of [3]. The proof is completed by a straightforward change of variables.

(c) The first part follows by letting $a \rightarrow \infty$ in (b), and the second part follows by looking at the complement and then using a similar argument as in (a).

\section{Creeping}

In principle, there are two ways for $U$ to enter $(-\infty, 0)$ continuously; either it goes below 0 by creeping during an excursion away from the curve $\bar{\gamma}$ or it creeps over 0 whilst moving along the curve $\bar{\gamma}$ at the moment that $\bar{\gamma}=0$ (see Figure 2). This leads to the next definition. 

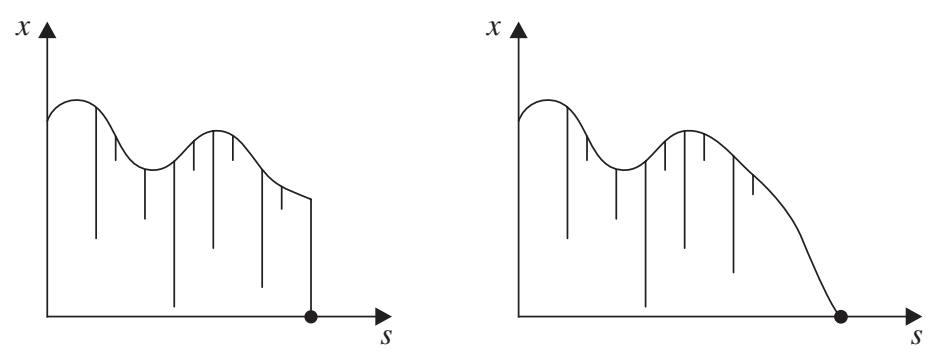

Figure 2: Two different ways for $U$ to creep into $(-\infty, 0)$.

Definition 3.1. We say that the process $U$ exhibits type-I creeping under $\mathrm{P}_{x}$ if $\mathrm{P}_{x}\left(U_{T_{0}^{-}}=0\right.$; $\left.\sigma_{a^{*}(x)}>T_{0}^{-}\right)>0$. We say that the process $U$ exhibits type-II creeping under $\mathrm{P}_{x}$ if $a^{*}(x)<\infty$ and $\mathrm{P}_{x}\left[\sigma_{a^{*}(x)}=T_{0}^{-}\right]>0$. Note that necessarily $U_{T_{0}^{-}}=0$ on the event $\left\{\sigma_{a^{*}(x)}=T_{0}^{-}\right\}$.

In Section 4 we will make some remarks regarding type-I creeping. However, for the present, let us dwell on type-II creeping, for which there exists an integral test.

Corollary 3.1. Fix $x>0$, and recall that $a^{*}(x)=\inf \{s \in[x, \infty): \bar{\gamma}(s)<0\}$. Assume that $a^{*}(x)<\infty$. We have, for all $q \geq 0$,

$$
\mathrm{E}_{x}\left[\mathrm{e}^{-q T_{0}^{-}} \mathbf{1}_{\left\{\sigma_{a^{*}(x)}=T_{0}^{-}\right\}}\right]=\exp \left(-\int_{x}^{a^{*}(x)} \frac{W^{(q) \prime}(\bar{\gamma}(s))}{W^{(q)}(\bar{\gamma}(s))} \mathrm{d} s\right) .
$$

If $X$ is a compound Poisson process then the time $X$ spends at the maximum has strictly positive Lebesgue measure and, hence, we would intuitively expect that type-II creeping occurs. In fact, under some assumptions on the behavior of $\bar{\gamma}$, it turns out that only spectrally negative Lévy processes of bounded variation possess the type-II creeping property.

Corollary 3.2. Fix $x>0$, and assume that $\gamma:[0, \infty) \rightarrow(1, \infty)$ is continuous. Furthermore, suppose that $a^{*}(x)<\infty$. Then $X$ exhibits type-II creeping under $\mathrm{P}_{x}$ if and only if $X$ is of bounded variation.

Proof. First observe that the assumptions on $\gamma$ imply that $\bar{\gamma}:\left(x, a^{*}(x)\right) \rightarrow(0, x)$ is a continuously differentiable bijection. Furthermore, let $C_{1}:=\min _{0 \leq s \leq a^{*}(x)}|1-\gamma(s)|>0$ and $C_{2}:=\max _{0 \leq s \leq a^{*}(x)}|1-\gamma(s)|<\infty$.

If $X$ is of bounded variation, and, hence, takes the form (1.1), we have, by a change of variables and (1.3),

$$
\begin{aligned}
\int_{x}^{a^{*}(x)} \frac{W^{\prime}(\bar{\gamma}(s))}{W(\bar{\gamma}(s))} \mathrm{d} s & =\int_{0}^{x} \frac{W^{\prime}(t)}{W(t)\left|1-\gamma\left(\bar{\gamma}^{-1}(t)\right)\right|} \mathrm{d} t \\
& \leq \frac{1}{C_{1}} \int_{0}^{x} \log (W)^{\prime}(t) \mathrm{d} t \\
& =\frac{1}{C_{1}}\left[\log (W(x))-\log \left(\frac{1}{\mathrm{~d}}\right)\right]
\end{aligned}
$$

and, hence, type-II creeping follows. On the other hand, if $X$ is of unbounded variation, it follows similarly that

$$
\int_{x}^{a^{*}(x)} \frac{W^{\prime}(\bar{\gamma}(s))}{W(\bar{\gamma}(s))} \mathrm{d} s \geq \frac{1}{C_{2}} \int_{0}^{x} \log (W)^{\prime}(t) \mathrm{d} t \geq \frac{1}{C_{2}}[\log (W(x))-\log (W(0+))] .
$$

The last expression equals $\infty$ since $W(0+)=0$ (see (1.3)) and, consequently, type-II creeping cannot occur. 
We conclude this section with an example of type-II creeping for a process $X$ which includes a Gaussian component $\sigma>0$ in the case that $\bar{\gamma}$ has infinite gradient when hitting 0 . This shows that relaxing the conditions on $\gamma$ can lead to type-II creeping in the unbounded variation case. To this end, we need some auxiliary quantities. Let $a>0$ be fixed, and define, for $y \in[0, a]$,

$$
f(y):=y-(a-y)^{1 / 2} \text {. }
$$

Clearly, $f(0)<0$ and $f(a)>0$. Since $f$ is strictly increasing on $[0, a]$ and continuous, by the intermediate value theorem, there exists a unique $x^{*} \in(0, a)$ such that $f\left(x^{*}\right)=0$. This $x^{*}$ will now serve as a starting point for $X$. Additionally, let $\gamma \in(1, \infty)$ and define, for $s \in[0, \infty)$,

$$
\gamma(s):= \begin{cases}1+\frac{1}{2}(a-s)^{-1 / 2}, & s \leq a, \\ \gamma, & s>a .\end{cases}
$$

Hence, using the definition of $x^{*}$, we see that

$$
\bar{\gamma}(s)= \begin{cases}(a-s)^{1 / 2}, & s \leq a, \\ (1-\gamma)(s-a), & s>a .\end{cases}
$$

In particular, $a^{*}\left(x^{*}\right)=a$. Changing variables and using the fact that $\sigma>0$ (which implies that $W \in C^{1}(0, \infty)$ and $\left.\lim _{u \downarrow 0} u W(u)^{-1}=\sigma^{2} / 2\right)$ yields

$$
\int_{x^{*}}^{a} \frac{W^{\prime}(\bar{\gamma}(s))}{W(\bar{\gamma}(s))} \mathrm{d} s=2 \int_{0}^{x^{*}} \frac{u W^{\prime}(u)}{W(u)} \mathrm{d} u \leq 2 x^{*} \sup _{0<u \leq x^{*}} \frac{u W^{\prime}(u)}{W(u)}<\infty .
$$

Hence, type-II creeping occurs under $\mathrm{P}_{x^{*}}$.

\section{Additional results relevant to risk theory}

Let us return to the setting of the stochastic perturbation $U$ in the setting of insurance risk. It is also possible to obtain the analogous statements to Theorems 1.2 and 1.3 of [7]. The analogue of the first of these two theorems concerns the expectation of a path functional which can be interpreted as the net present value of tax paid until ruin and reads as follows.

Theorem 4.1. Let $x>0$, and recall that $a^{*}(x)=\inf \{s \in[x, \infty): \bar{\gamma}(s)<0\} \in(0, \infty]$. For $q \geq 0$, we have

$$
\mathrm{E}_{x}\left[\int_{0}^{T_{0}^{-}} \mathrm{e}^{-q u} \gamma\left(S_{u}\right) \mathrm{d} S_{u}\right]=\int_{x}^{a^{*}(x)} \exp \left(-\int_{x}^{t} \frac{W^{(q) \prime}(\bar{\gamma}(s))}{W^{(q)}(\bar{\gamma}(s))} \mathrm{d} s\right) \gamma(t) \mathrm{d} t .
$$

Proof. For $t \geq 0$, let $S_{t}^{-1}:=\inf \left\{u>0: S_{u}>t\right\}$ be the right inverse of $S$ and note that, with probability 1 , the functions $t \mapsto S_{t}^{-1}$ and $t \mapsto \sigma_{t}$ agree almost everywhere. Then, similarly to the proof of Theorem 1.2 of [7], changing variables and applying Fubini's theorem gives

$$
\begin{aligned}
\mathrm{E}_{x}\left[\int_{0}^{T_{0}^{-}} \mathrm{e}^{-q u} \gamma\left(S_{u}\right) \mathrm{d} S_{u}\right] & =\mathrm{E}_{x}\left[\int_{0}^{\infty} \mathbf{1}_{\left\{u<T_{0}^{-}\right\}} \mathrm{e}^{-q u} \gamma\left(S_{u}\right) \mathrm{d} S_{u}\right] \\
& =\mathrm{E}_{x}\left[\int_{0}^{\infty} \mathbf{1}_{\left\{S_{t}^{-1}<T_{0}^{-}\right\}} \mathrm{e}^{-q S_{t}^{-1}} \gamma(t) \mathrm{d} t\right] \\
& =\mathrm{E}_{x}\left[\int_{0}^{\infty} \mathbf{1}_{\left\{\sigma_{t}<T_{0}^{-}\right\}} \mathrm{e}^{-q \sigma_{t}} \gamma(t) \mathrm{d} t\right] \\
& =\int_{x}^{a^{*}(x)} \mathrm{E}_{x}\left[{ }^{-q \sigma_{t}} \mathbf{1}_{\left\{\sigma_{t}<T_{0}^{-}\right\}}\right] \gamma(t) \mathrm{d} t .
\end{aligned}
$$

Replacing the expectation with the expression in Theorem 2.1(a) completes the proof. 
Remark 4.1. Fix $x>0$, and suppose that $\gamma(s)=\gamma \in(1, \infty)$. A computation as in Remark 2.2 shows that

$$
\mathrm{E}_{x}\left[\int_{0}^{T_{0}^{-}} \mathrm{e}^{-q u} \gamma\left(S_{u}\right) \mathrm{d} S_{u}\right]=\frac{\gamma}{\gamma-1} \int_{0}^{x}\left(\frac{W^{(q)}(t)}{W^{(q)}(x)}\right)^{1 /(\gamma-1)} \mathrm{d} t .
$$

Similarly, if we assume that $\gamma(s) \equiv \gamma \in(0,1)$, it is straightforward to recover the second formula in Remark 1.1 of [7].

Although unnecessary, for the sake of presentational convenience, we will restrict ourselves to the case that $\gamma:[0, \infty) \rightarrow(1, \infty)$ in order to state an analogue of Theorem 1.3 of [7]. In that case, $\bar{\gamma}$ is a strictly decreasing function and accordingly has an inverse, $\bar{\gamma}^{-1}$. Note that in [7] it was assumed that $\gamma:[0, \infty) \rightarrow[0,1)$ such that $\int_{0}^{\infty}(1-\gamma(s)) \mathrm{d} s=\infty$. If we refer to the latter as a light tax regime then we may think of the current setting as a heavy tax regime. We have the following result, the second part of which addresses the issue of type-I creeping.

Theorem 4.2. Fix $x>0$, and suppose that $a^{*}(x)<\infty$. Let $\kappa:=L_{L_{T_{0}^{-}}^{-1}}^{-1}$, the last moment that tax is paid before ruin. Denote by $v$ the Lévy measure of $-X$. For any $z>0, x>\theta \geq y \geq 0$, and $\alpha, \beta \geq 0$, we have

$$
\begin{aligned}
\mathrm{E}_{x}\left[\mathrm{e}^{-\alpha \kappa-\beta\left(T_{0}^{-}-\kappa\right)} ; A_{T_{0}^{-}} \in \mathrm{d} \theta, U_{T_{0}^{-}-} \in \mathrm{d} y,-U_{T_{0}^{-}} \in \mathrm{d} z\right] \\
=\frac{1}{\gamma\left(\bar{\gamma}^{-1}(\theta)\right)-1} \exp \left(-\int_{\theta}^{x} \frac{W^{(\alpha) \prime}(v)}{W^{(\alpha)}(v)\left(\gamma\left(\bar{\gamma}^{-1}(v)\right)-1\right)} \mathrm{d} v\right) \\
\times\left[\left\{W^{(\beta)^{\prime}}(\theta-y)-\frac{W^{(\beta)^{\prime}}(\theta)}{W^{(\beta)}(\theta)} W^{(\beta)}(\theta-y)\right\} v(y+\mathrm{d} z) \mathbf{1}_{\{y<\theta\}} \mathrm{d} y\right. \\
\left.\quad+W^{(\beta)}(0+) v(\theta+\mathrm{d} z) \delta_{\theta}(\mathrm{d} y)\right] \mathrm{d} \theta,
\end{aligned}
$$

where $\delta_{\theta}(\mathrm{d} y)$ is the Dirac measure which assigns unit mass to the point $\theta$. Furthermore, for $0<\theta<x$, we also have

$$
\begin{aligned}
\mathrm{E}_{x}\left[\mathrm{e}^{-\alpha \kappa-\beta\left(T_{0}^{-}-\kappa\right)} ; A_{T_{0}^{-}} \in \mathrm{d} \theta, U_{T_{0}^{-}}=0\right] \\
=\frac{1}{\gamma\left(\bar{\gamma}^{-1}(\theta)\right)-1} \exp \left(-\int_{\theta}^{x} \frac{W^{(\alpha) \prime}(y)}{W^{(\alpha)}(y)\left(\gamma\left(\bar{\gamma}^{-1}(y)\right)-1\right)} \mathrm{d} y\right) \\
\quad \times \frac{\sigma^{2}}{2}\left\{\frac{W^{(\beta) \prime}(\theta)^{2}}{W^{(\beta)}(\theta)}-W^{(\beta) \prime \prime}(\theta)\right\} \mathrm{d} \theta,
\end{aligned}
$$

where $\sigma$ is the Gaussian coefficient in the Lévy-Itô decomposition.

The proof of this theorem is virtually identical to the proof of Theorem 1.3 of [7] once the obvious adjustments have been made and is thus left as an exercise to the reader.

\section{Acknowledgement}

We would like to thank an anonymous referee for their valuable comments which led to improvements in this paper. 


\section{References}

[1] Albrecher, H. AND HiPP, C. (2007). Lundberg's risk process with tax. Blätter DGVFM 28, 13-28.

[2] Albrecher, H., Renaud, J.-F. and Zhou, X. (2008). A Lévy insurance risk process with tax. J. Appl. Prob. 45, 363-375.

[3] Avram, F., Kyprianou, A. E. and Pistorius, M. R. (2004). Exit problems for spectrally negative Lévy processes and applications to (Canadized) Russian options. Ann. Appl. Prob. 14, 215-238.

[4] Bertoin, J. (1996). Lévy Processes. Cambrdige University Press.

[5] Bichteler, K. (2002). Stochastic Integration with Jumps. Cambridge University Press.

[6] Kuznetsov, A., Kyprianou, A. E. And Rivero, V. (2012). The theory of scale functions for spectrally negative Lévy processes. In Lévy Matters II (Lecture Notes Math. 2061), Springer, Berlin, pp. 97-186.

[7] Kyprianou, A. E. And Zhou, X. (2009). General tax structures and the Lévy insurance risk model. J. Appl. Prob. 46, 1146-1156.

[8] Kyprianou, A. E. (2006). Introductory Lectures on Fluctuations of Lévy Processes with Applications. Springer, Berlin.

[9] Perman, M. and Werner, W. (1997). Perturbed Brownian motions. Prob. Theory Relat. Fields 108, $357-383$. 